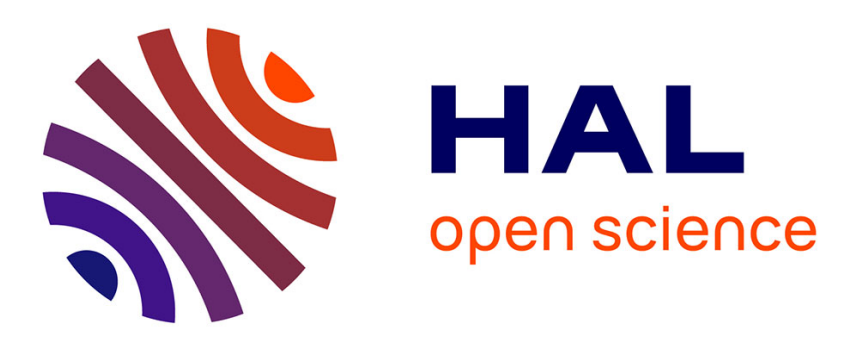

\title{
Completely monotonic degrees for a difference between the logarithmic and psi functions \\ Feng Qi, Ai-Qi Liu
}

\section{To cite this version:}

Feng Qi, Ai-Qi Liu. Completely monotonic degrees for a difference between the logarithmic and psi functions: A difference between logarithmic and psi functions. Journal of Computational and Applied Mathematics, 2019, 361, pp.366-371. 10.1016/j.cam.2019.05.001 . hal-01728682v2

\section{HAL Id: hal-01728682 \\ https://hal.science/hal-01728682v2}

Submitted on 19 Jan 2020

HAL is a multi-disciplinary open access archive for the deposit and dissemination of scientific research documents, whether they are published or not. The documents may come from teaching and research institutions in France or abroad, or from public or private research centers.
L'archive ouverte pluridisciplinaire HAL, est destinée au dépôt et à la diffusion de documents scientifiques de niveau recherche, publiés ou non, émanant des établissements d'enseignement et de recherche français ou étrangers, des laboratoires publics ou privés. 


\title{
COMPLETELY MONOTONIC DEGREES FOR A DIFFERENCE BETWEEN THE LOGARITHMIC AND PSI FUNCTIONS
}

\author{
FENG QI AND AI-QI LIU
}

\begin{abstract}
In the paper, the authors firstly present a concise proof for complete monotonicity of a function involving a difference between the logarithmic and psi functions, secondly compute completely monotonic degree of the above-mentioned function, and finally pose several conjectures on completely monotonic degrees of remainders and their derivatives for the asymptotic formula of the logarithm of the classical Euler gamma function.
\end{abstract}

\section{Preliminaries}

Recall from [16, Chapter XIII], [31, Chapter 1], and [32, Chapter IV] that a function $f$ is said to be completely monotonic on an interval $I$ if $f$ has derivatives of all orders on $I$ and

$$
(-1)^{n} f^{(n)}(x) \geq 0, \quad x \in I, \quad n \in\{0\} \cup \mathbb{N} .
$$

Theorem 12b in [32, p. 161] states that a necessary and sufficient condition for $f(x)$ to be completely monotonic on $(0, \infty)$ is that

$$
f(x)=\int_{0}^{\infty} e^{-x t} \mathrm{~d} \mu(t), \quad x \in(0, \infty),
$$

where $\mu(t)$ is non-decreasing and the above integral converges for $x \in(0, \infty)$. In other words or simply speaking, a function is completely monotonic on $(0, \infty)$ if and only if it is a Laplace transform of a non-negative measure.

In [2, pp. 374-375, Theorem 1] and [8, Theorem 1], it was verified that the function

$$
x^{\alpha}[\ln x-\psi(x)], \quad \alpha \in \mathbb{R}
$$

is completely monotonic on $(0, \infty)$ if and only if $\alpha \leq 1$, where $\psi(z)=\frac{\Gamma^{\prime}(z)}{\Gamma(z)}$ is called the psi function and $\Gamma(z)$ denotes the classical Euler gamma function which can be defined [1, 8, 18, 30, by

$$
\Gamma(z)=\int_{0}^{\infty} t^{z-1} e^{-t} \mathrm{~d} t, \quad \Re(z)>0
$$

2010 Mathematics Subject Classification. Primary 26A48; Secondary 33B15, 44A10.

Key words and phrases. complete monotonicity; completely monotonic degree; difference; psi function; logarithmic function; concise proof; gamma function; asymptotic formula; remainder; conjecture.

Please cite this article as "Feng Qi and Ai-Qi Liu, Completely monotonic degrees for a difference between the logarithmic and psi functions, Journal of Computational and Applied Mathematics 361 (2019), 366-371; available online at https://doi.org/10.1016/j.cam.2019.05.001" 
or by

$$
\Gamma(z)=\lim _{n \rightarrow \infty} \frac{n ! n^{z}}{\prod_{k=0}^{n}(z+k)}, \quad z \in \mathbb{C} \backslash\{0,-1,-2, \ldots\} .
$$

Let $f(x)$ be a completely monotonic function on $(0, \infty)$ and denote $f(\infty)=$ $\lim _{x \rightarrow \infty} f(x) \geq 0$. When the function $x^{\alpha}[f(x)-f(\infty)]$ is completely monotonic on $(0, \infty)$ if and only if $0 \leq \alpha \leq r \in \mathbb{R}$, we say that the number $r$ is the completely monotonic degree of $f(x)$ with respect to $x \in(0, \infty)$; if the function $x^{\alpha}[f(x)-f(\infty)]$ is completely monotonic on $(0, \infty)$ for all $\alpha \in \mathbb{R}$, then we say that the completely monotonic degree of $f(x)$ with respect to $x \in(0, \infty)$ is $\infty$. For convenience, a notation $\operatorname{deg}_{\mathrm{cm}}^{x}[f(x)]$ was designed to denote the completely monotonic degree $r$ of $f(x)$ with respect to $x \in(0, \infty)$. For more information on completely monotonic degree and its properties, please refer to the papers $44,10,12,13,14,15,19,20$, $23,24,25,26,28,29$, and closely related references therein.

The necessary and sufficient condition $\alpha \leq 1$ for the function (2) to be completely monotonic on $(0, \infty)$ means that the completely monotonic degree of the completely monotonic function $\ln x-\psi(x)$ on $(0, \infty)$ is

$$
\operatorname{deg}_{\mathrm{cm}}^{x}[\ln x-\psi(x)]=1 .
$$

In [30, Theorem 1.7], it was proved that the function

$$
x^{2}[\psi(x)-\ln x]+\frac{x}{2}
$$

is strictly decreasing and convex on $(0, \infty)$ and, as $x \rightarrow \infty$, tends to $-\frac{1}{12}$. In $[3$, Theorem 1], it was discovered that the function

$$
\Phi(x)=x^{2}[\psi(x)-\ln x]+\frac{x}{2}+\frac{1}{12}
$$

is completely monotonic on $(0, \infty)$.

In the second section of this paper, motivated by the second proof of 8 , Theorem 1] and with the aid of some conclusions in [33, we will present a concise proof, which is simpler and shorter than the corresponding proof in [3, Theorem 1], for complete monotonicity of the function $\Phi(x)$ on $(0, \infty)$.

In the third section of this paper, we will compute the completely monotonic degree of the completely monotonic function

$$
\phi(x)=\frac{\Phi(x)}{x^{2}}=\psi(x)-\ln x+\frac{1}{2 x}+\frac{1}{12 x^{2}}
$$

on $(0, \infty)$.

In the fourth section of this paper, we will pose conjectures on completely monotonic degrees of remainders for the asymptotic formula of $\ln \Gamma(x)$ and for polygamma functions $\psi^{(k)}(x)$ for $k \geq 0$.

\section{Complete monotonicity of the function $\Phi(x)$}

The first main result in this paper can be stated as the following theorem.

Theorem 1. The function $\Phi(x)$ defined in (3) is completely monotonic on $(0, \infty)$, with the limits

$$
\lim _{x \rightarrow 0^{+}} \Phi(x)=\frac{1}{12} \quad \text { and } \quad \lim _{x \rightarrow \infty} \Phi(x)=0 .
$$


Proof. In [18, p. 140, 5.9.13], it was listed that

$$
\psi(z)=\ln z+\int_{0}^{\infty}\left(\frac{1}{t}-\frac{1}{1-e^{-t}}\right) e^{-t z} \mathrm{~d} t .
$$

By integration by parts, this formula can be reformulated as

$$
\begin{aligned}
\psi(x)- & \ln x+\frac{1}{x}=\int_{0}^{\infty}\left(\frac{1}{t}-\frac{1}{e^{t}-1}\right) e^{-x t} \mathrm{~d} t \\
& =-\frac{1}{x}\left\{\left.\left[h(t) e^{-x t}\right]\right|_{t=0} ^{t=\infty}-\int_{0}^{\infty} h^{\prime}(t) e^{-x t} \mathrm{~d} t\right\}=\frac{1}{2 x}+\frac{1}{x} \int_{0}^{\infty} h^{\prime}(t) e^{-x t} \mathrm{~d} t,
\end{aligned}
$$

where the function

$$
h(t)= \begin{cases}\frac{1}{t}-\frac{1}{e^{t}-1}, & t \neq 0 \\ \frac{1}{2}, & t=0\end{cases}
$$

is convex on $(0, \infty)$, with the limits

$$
\lim _{t \rightarrow 0^{+}} h^{\prime}(t)=-\frac{1}{12} \text { and } \lim _{t \rightarrow \infty} h^{\prime}(t)=0 .
$$

For detailed information on the function $h(t)$, please refer to [5, 6, 7, 21 and closely related references therein. Consequently, integrating by parts again yields

$$
\Phi(x)=\frac{1}{12}+x \int_{0}^{\infty} h^{\prime}(t) e^{-x t} \mathrm{~d} t=\frac{1}{12}-\int_{0}^{\infty} h^{\prime}(t) \mathrm{d}\left(e^{-x t}\right)=\int_{0}^{\infty} h^{\prime \prime}(t) e^{-x t} \mathrm{~d} t .
$$

Accordingly, by virtue of [32, p. 161, Theorem 12b] mentioned at the beginning of this paper and with the help of the convexity for the function $h(t)$ on $(0, \infty)$, we immediately see that the function $\Phi(x)$ is completely monotonic on $(0, \infty)$, while, in light of the limits in (6), we readily derive the limits in (5). The proof of Theorem 1 is complete.

\section{Completely monotonic Degree of the Function $\phi(x)$}

The second main result in this paper can be stated as the following theorem.

Theorem 2. The completely monotonic degree of the completely monotonic function $\phi(x)$ defined in (4) on $(0, \infty)$ is

$$
\operatorname{deg}_{\mathrm{cm}}^{x}[\phi(x)]=2 .
$$

Proof. Since the relation $\Phi(x)=x^{2} \phi(x)$ and the function $\phi(x)$ is completely monotonic on $(0, \infty)$, see [22, Theorem 1], then Theorem 1 in [3] and Theorem 1 in this paper mean

$$
\operatorname{deg}_{\mathrm{cm}}^{x}[\phi(x)] \geq 2 .
$$

On the other hand side, if $x^{\alpha} \phi(x)$ is completely monotonic on $(0, \infty)$, then its first derivative is not positive, that is,

$$
\alpha x^{\alpha-1} \phi(x)+x^{\alpha} \phi^{\prime}(x) \leq 0
$$

which can be rearranged as

$$
\alpha \leq-\frac{x \phi^{\prime}(x)}{\phi(x)}=-\frac{x^{3}\left[\psi^{\prime}(x+1)-\frac{1}{x}+\frac{1}{2 x^{2}}-\frac{1}{6 x^{3}}\right]}{x^{2}[\psi(x)-\ln x]+\frac{x}{2}+\frac{1}{12}} \rightarrow 2
$$


for $x \rightarrow 0^{+}$, where we used the recursion relation $\psi^{\prime}(x+1)=\psi^{\prime}(x)-\frac{1}{x^{2}}$ and the first limit in (5). This means that

$$
\operatorname{deg}_{\mathrm{cm}}^{x}[\phi(x)] \leq 2 .
$$

Combining (8) with (9) concludes that the completely monotonic degree of the function $\phi(x)$ on $(0, \infty)$ is 2 . The proof of Theorem 2 is complete.

\section{Conjectures}

The third main result in this paper is to pose conjectures on completely monotonic degrees of remainders for the asymptotic formula of $\ln \Gamma(x)$ and on completely monotonic degrees of derivatives of these remainders.

In [22, Theorem 1], among other things, the function $\phi(x)$ and three functions

$$
\ln x-\frac{1}{2 x}-\psi(x), \quad \psi^{\prime}(x)-\frac{1}{x}-\frac{1}{2 x^{2}}-\frac{1}{6 x^{3}}+\frac{1}{30 x^{5}}, \quad \frac{1}{x}+\frac{1}{2 x^{2}}+\frac{1}{6 x^{3}}-\psi^{\prime}(x)
$$

were proved to be completely monotonic on $(0, \infty)$. What are the completely monotonic degrees of these three functions on $(0, \infty)$ ? We guess that

$$
\begin{aligned}
\operatorname{deg}_{\mathrm{cm}}^{x}\left[\ln x-\frac{1}{2 x}-\psi(x)\right] & =1, \\
\operatorname{deg}_{\mathrm{cm}}^{x}\left[\frac{1}{x}+\frac{1}{2 x^{2}}+\frac{1}{6 x^{3}}-\psi^{\prime}(x)\right] & =2, \\
\operatorname{deg}_{\mathrm{cm}}^{x}\left[\psi^{\prime}(x)-\frac{1}{x}-\frac{1}{2 x^{2}}-\frac{1}{6 x^{3}}+\frac{1}{30 x^{5}}\right] & =4 .
\end{aligned}
$$

In [2, Theorem 8] and [11, Theorem 2], the functions

$$
R_{n}(x)=(-1)^{n}\left[\ln \Gamma(x)-\left(x-\frac{1}{2}\right) \ln x+x-\frac{1}{2} \ln (2 \pi)-\sum_{k=1}^{n} \frac{B_{2 k}}{(2 k-1) 2 k} \frac{1}{x^{2 k-1}}\right]
$$

for $n \geq 0$ were proved to be completely monotonic on $(0, \infty)$. Consequently, the functions $(-1)^{m}\left[R_{n}(x)\right]^{(m)}$ for $m, n \geq 0$ are completely monotonic on $(0, \infty)$. See also [9, Section 1.4] and [17, Theorem 3.1]. The functions $(-1)^{n} R_{n}(x)$ for $n \geq 0$ are remainders for the asymptotic formula of $\ln \Gamma(x)$. See [1, p. 257, 6.1.40] and [18, p. $140,5.11 .1$.

In [15. Theorem 2.1], the completely monotonic degree of the function $R_{n}(x)$ for $n \geq 0$ on $(0, \infty)$ was proved to be at least $n$. The equations $(7),(10),(11)$, and $(12)$ and plotting by the well-known software Wolfram Mathematica imply that

$$
\begin{array}{ll}
\operatorname{deg}_{\mathrm{cm}}^{x}\left[(-1)^{0}\left[R_{0}(x)\right]^{(0)}\right]=0, & \operatorname{deg}_{\mathrm{cm}}^{x}\left[(-1)^{0}\left[R_{1}(x)\right]^{(0)}\right]=1, \\
\operatorname{deg}_{\mathrm{cm}}^{x}\left[(-1)^{0}\left[R_{2}(x)\right]^{(0)}\right]=2, & \operatorname{deg}_{\mathrm{cm}}^{x}\left[(-1)^{0}\left[R_{3}(x)\right]^{(0)}\right]=4, \\
\operatorname{deg}_{\mathrm{cm}}^{x}\left[(-1)^{0}\left[R_{4}(x)\right]^{(0)}\right]=6, & \operatorname{deg}_{\mathrm{cm}}^{x}\left[(-1)^{0}\left[R_{5}(x)\right]^{(0)}\right]=8, \\
\operatorname{deg}_{\mathrm{cm}}^{x}\left[(-1)^{1}\left[R_{0}(x)\right]^{(1)}\right]=1, & \operatorname{deg}_{\mathrm{cm}}^{x}\left[(-1)^{1}\left[R_{1}(x)\right]^{(1)}\right]=2, \\
\operatorname{deg}_{\mathrm{cm}}^{x}\left[(-1)^{1}\left[R_{2}(x)\right]^{(1)}\right]=3, & \operatorname{deg}_{\mathrm{cm}}^{x}\left[(-1)^{1}\left[R_{3}(x)\right]^{(1)}\right]=5, \\
\operatorname{deg}_{\mathrm{cm}}^{x}\left[(-1)^{1}\left[R_{4}(x)\right]^{(1)}\right]=7, & \operatorname{deg}_{\mathrm{cm}}^{x}\left[(-1)^{1}\left[R_{5}(x)\right]^{(1)}\right]=9, \\
\operatorname{deg}_{\mathrm{cm}}^{x}\left[(-1)^{2}\left[R_{0}(x)\right]^{(2)}\right]=1, & \operatorname{deg}_{\mathrm{cm}}^{x}\left[(-1)^{2}\left[R_{1}(x)\right]^{(2)}\right]=2, \\
\operatorname{deg}_{\mathrm{cm}}^{x}\left[(-1)^{2}\left[R_{2}(x)\right]^{(2)}\right]=4, & \operatorname{deg}_{\mathrm{cm}}^{x}\left[(-1)^{2}\left[R_{3}(x)\right]^{(2)}\right]=6,
\end{array}
$$




$$
\begin{array}{rlrl}
\operatorname{deg}_{\mathrm{cm}}^{x}\left[(-1)^{2}\left[R_{4}(x)\right]^{(2)}\right] & =8, & & \operatorname{deg}_{\mathrm{cm}}^{x}\left[(-1)^{2}\left[R_{5}(x)\right]^{(2)}\right]=10, \\
\operatorname{deg}_{\mathrm{cm}}^{x}\left[(-1)^{3}\left[R_{0}(x)\right]^{(3)}\right] & =2, & & \operatorname{deg}_{\mathrm{cm}}^{x}\left[(-1)^{3}\left[R_{1}(x)\right]^{(3)}\right]=3, \\
\operatorname{deg}_{\mathrm{cm}}^{x}\left[(-1)^{3}\left[R_{2}(x)\right]^{(3)}\right] & =5, & & \operatorname{deg}_{\mathrm{cm}}^{x}\left[(-1)^{3}\left[R_{3}(x)\right]^{(3)}\right]=7, \\
\operatorname{deg}_{\mathrm{cm}}^{x}\left[(-1)^{3}\left[R_{4}(x)\right]^{(3)}\right] & =9, & & \operatorname{deg}_{\mathrm{cm}}^{x}\left[(-1)^{3}\left[R_{5}(x)\right]^{(3)}\right]=11, \\
\operatorname{deg}_{\mathrm{cm}}^{x}\left[(-1)^{4}\left[R_{0}(x)\right]^{(4)}\right]=3, & & \operatorname{deg}_{\mathrm{cm}}^{x}\left[(-1)^{4}\left[R_{1}(x)\right]^{(4)}\right]=4, \\
\operatorname{deg}_{\mathrm{cm}}^{x}\left[(-1)^{4}\left[R_{2}(x)\right]^{(4)}\right] & =6, & & \operatorname{deg}_{\mathrm{cm}}^{x}\left[(-1)^{4}\left[R_{3}(x)\right]^{(4)}\right]=8, \\
\operatorname{deg}_{\mathrm{cm}}^{x}\left[(-1)^{4}\left[R_{4}(x)\right]^{(4)}\right]=10, & \operatorname{deg}_{\mathrm{cm}}^{x}\left[(-1)^{4}\left[R_{5}(x)\right]^{(4)}\right]=12, \\
\operatorname{deg}_{\mathrm{cm}}^{x}\left[(-1)^{5}\left[R_{0}(x)\right]^{(5)}\right] & =4, & \operatorname{deg}_{\mathrm{cm}}^{x}\left[(-1)^{5}\left[R_{1}(x)\right]^{(5)}\right] & =5, \\
\operatorname{deg}_{\mathrm{cm}}^{x}\left[(-1)^{5}\left[R_{2}(x)\right]^{(5)}\right] & =7, & & \operatorname{deg}_{\mathrm{cm}}^{x}\left[(-1)^{5}\left[R_{3}(x)\right]^{(5)}\right]=9, \\
\operatorname{deg}_{\mathrm{cm}}^{x}\left[(-1)^{5}\left[R_{4}(x)\right]^{(5)}\right] & =11, & & \operatorname{deg}_{\mathrm{cm}}^{x}\left[(-1)^{5}\left[R_{5}(x)\right]^{(5)}\right]=13 .
\end{array}
$$

What are the completely monotonic degrees of the completely monotonic functions $(-1)^{m}\left[R_{n}(x)\right]^{(m)}$ for $m, n \geq 0$ on $(0, \infty)$ ? We conjecture that

(i) when $m=0$, the completely monotonic degrees of $R_{n}(x)$ on $(0, \infty)$ satisfy

$$
\operatorname{deg}_{\mathrm{cm}}^{x}\left[R_{0}(x)\right]=0, \quad \operatorname{deg}_{\mathrm{cm}}^{x}\left[R_{1}(x)\right]=1,
$$

and

$$
\operatorname{deg}_{\text {cm }}^{x}\left[R_{n}(x)\right]=2(n-1), \quad n \geq 2 ;
$$

(ii) when $m=1$, the completely monotonic degrees of $-R_{n}^{\prime}(x)$ on $(0, \infty)$ satisfy

$$
\operatorname{deg}_{\mathrm{cm}}^{x}\left[-R_{0}^{\prime}(x)\right]=1, \quad \operatorname{deg}_{\mathrm{cm}}^{x}\left[-R_{1}^{\prime}(x)\right]=2,
$$

and

$$
\operatorname{deg}_{\mathrm{cm}}^{x}\left[-R_{n}^{\prime}(x)\right]=2 n-1, \quad n \geq 2 ;
$$

(iii) when $m \geq 2$, the completely monotonic degrees of $(-1)^{m}\left[R_{n}(x)\right]^{(m)}$ satisfy $\operatorname{deg}_{\text {cm }}^{x}\left[(-1)^{m}\left[R_{0}(x)\right]^{(m)}\right]=m-1, \quad \operatorname{deg}_{\text {cm }}^{x}\left[(-1)^{m}\left[R_{1}(x)\right]^{(m)}\right]=m$,

and

$$
\operatorname{deg}_{\mathrm{cm}}^{x}\left[(-1)^{m}\left[R_{n}(x)\right]^{(m)}\right]=m+2(n-1), \quad n \geq 2 .
$$

\section{REMARKS}

Finally we give several remarks on the significance and importance of results obtained in this paper.

Remark 1. The significance of completely monotonic functions can be seen from the above equation (1) cited from [32, Theorem 12b].

Completely monotonic degree is a new notion introduced in very recent years. See 44, 10, 12, 13, 14, 15, 19, 20, 23, 24, 25, 26, 28, 29 and closely related references. This new notion can be used to measure and differentiate complete monotonicity more accurately. For example, the functions $\frac{1}{x^{\alpha}}$ and $\frac{1}{x^{\beta}}$ for $\alpha, \beta>0$ and $\alpha \neq \beta$ are both completely monotonic on $(0, \infty)$, but they are different completely monotonic functions. How to quantitatively measure their difference? How to quantitatively differentiate them? The notion of completely monotonic degrees is useful: the completely monotonic degrees of these functions $\frac{1}{x^{\alpha}}$ and $\frac{1}{x^{\beta}}$ for $\alpha, \beta>0$ and $\alpha \neq \beta$ are $\alpha>0$ and $\beta>0$ respectively. 
On the other hand, the classical gamma function $\Gamma(z)$ and polygamma functions $\psi^{(k)}$ for $k \geq 0$ and $x>0$ are fundamental in mathematics and mathematical sciences. The asymptotic expansions of $\ln \Gamma(z)$ and $\psi^{(k)}$ for $k \geq 0$ have extensive applications in almost all mathematical sciences. The remainders $(-1)^{m}\left[R_{n}(x)\right]^{(m)}$ for $m, n \geq 0$ in 13 have been proved to be completely monotonic on $(0, \infty)$ by several mathematicians in Germany, Denmark, Romania, Cyprus, China, and so on. How to quantitatively differentiate complete monotonicity of these remainders? This is the main contribution of this paper.

The functions $\ln x-\psi(x)$ and $\psi(x)-\ln x+\frac{1}{2 x}+\frac{1}{12 x^{2}}$, which are simpler but applied extensively, and their complete monotonicity on $(0, \infty)$ have been studied for many years by many mathematicians in many top mathematical journals. In this paper, we tried and succeeded to strengthen and differentiate these known conclusions.

What are motivations of advances for pure mathematics? Questions! Problems! Guesses! Conjectures! Hypotheses! Therefore, it is very important to pose correct and significant problems for pure mathematics. To pose correct and significant problems is difficult, even very difficult, because it needs deep and intelligent insight into mathematics. We believe that we posed correct and significant problems in this paper.

Remark 2. This paper is a largely expanded version of the preprint [27].

Acknowledgements. The authors are grateful to anonymous referees for their careful corrections to and valuable comments on the original version of this paper.

\section{REFERENCES}

[1] M. Abramowitz and I. A. Stegun (Eds), Handbook of Mathematical Functions with Formulas, Graphs, and Mathematical Tables, National Bureau of Standards, Applied Mathematics Series 55, 10th printing, Dover Publications, New York and Washington, 1972.

[2] H. Alzer, On some inequalities for the gamma and psi functions, Math. Comp. 66 (1997), no. 217, 373-389; available online at https://doi.org/10.1090/S0025-5718-97-00807-7

[3] C.-P. Chen, F. Qi, and H. M. Srivastava, Some properties of functions related to the gamma and psi functions, Integral Transforms Spec. Funct. 21 (2010), no. 2, 153-164; available online at https://doi.org/10.1080/10652460903064216

[4] B.-N. Guo and F. Qi, A completely monotonic function involving the tri-gamma function and with degree one, Appl. Math. Comput. 218 (2012), no. 19, 9890-9897; available online at https://doi.org/10.1016/j.amc.2012.03.075

[5] B.-N. Guo and F. Qi, A simple proof of logarithmic convexity of extended mean values, Numer. Algorithms 52 (2009), no. 1, 89-92; available online at https://doi.org/10.1007/ s11075-008-9259-7

[6] B.-N. Guo and F. Qi, Properties and applications of a function involving exponential functions, Commun. Pure Appl. Anal. 8 (2009), no. 4, 1231-1249; available online at https://doi.org/10.3934/cpaa.2009.8.1231

[7] B.-N. Guo and F. Qi, The function $\left(b^{x}-a^{x}\right) / x$ : Logarithmic convexity and applications to extended mean values, Filomat 25 (2011), no. 4, 63-73; available online at https://doi.org/ 10.2298/FIL1104063G

[8] B.-N. Guo and F. Qi, Two new proofs of the complete monotonicity of a function involving the psi function, Bull. Korean Math. Soc. 47 (2010), no. 1, 103-111; available online at https://doi.org/10.4134/bkms.2010.47.1.103

[9] B.-N. Guo, F. Qi, J.-L. Zhao, and Q.-M. Luo, Sharp inequalities for polygamma functions, Math. Slovaca 65 (2015), no. 1, 103-120; available online at https://doi.org/10.1515/ ms-2015-0010. 
[10] S. Koumandos, Monotonicity of some functions involving the gamma and psi functions, Math. Comp. 77 (2008), no. 264, 2261-2275; available online at https://doi.org/10.1090/ S0025-5718-08-02140-6

[11] S. Koumandos, Remarks on some completely monotonic functions, J. Math. Anal. Appl. 324 (2006), no. 2, 1458-1461; available online at http://dx.doi.org/10.1016/j.jmaa.2005.12. 017

[12] S. Koumandos and M. Lamprecht, Some completely monotonic functions of positive order, Math. Comp. 79 (2010), no. 271, 1697-1707; available online at https://doi.org/10.1090/ S0025-5718-09-02313-8

[13] S. Koumandos and M. Lamprecht, Complete monotonicity and related properties of some special functions, Math. Comp. 82 (2013), no. 282, 1097-1120; available online at https: //doi.org/10.1090/S0025-5718-2012-02629-9

[14] S. Koumandos and H. L. Pedersen, Absolutely monotonic functions related to Euler's gamma function and Barnes' double and triple gamma function, Monatsh. Math. 163 (2011), no. 1, 51-69; available online at https://doi.org/10.1007/s00605-010-0197-9.

[15] S. Koumandos and H. L. Pedersen, Completely monotonic functions of positive order and asymptotic expansions of the logarithm of Barnes double gamma function and Euler's gamma function, J. Math. Anal. Appl. 355 (2009), no. 1, 33-40; available online at https://doi. org/10.1016/j.jmaa.2009.01.042

[16] D. S. Mitrinović, J. E. Pečarić, and A. M. Fink, Classical and New Inequalities in Analysis, Kluwer Academic Publishers, Dordrecht-Boston-London, 1993; available online at https: //doi.org/10.1007/978-94-017-1043-5

[17] C. Mortici, Very accurate estimates of the polygamma functions, Asympt. Anal. 68 (2010), no. 3, 125-134; available online at http://dx.doi.org/10.3233/ASY-2010-0983.

[18] F. W. J. Olver, D. W. Lozier, R. F. Boisvert, and C. W. Clark (eds.), NIST Handbook of Mathematical Functions, Cambridge University Press, New York, 2010; available online at http://dlmf.nist.gov/

[19] F. Qi, Properties of modified Bessel functions and completely monotonic degrees of differences between exponential and trigamma functions, Math. Inequal. Appl. 18 (2015), no. 2, 493-518; available online at https://doi.org/10.7153/mia-18-37

[20] F. Qi and R. P. Agarwal, On complete monotonicity for several classes of functions related to ratios of gamma functions, J. Inequal. Appl. 2019, Paper No. 36, 42 pages; available online at https://doi.org/10.1186/s13660-019-1976-z

[21] F. Qi and R. J. Chapman, Two closed forms for the Bernoulli polynomials, J. Number Theory 159 (2016), 89-100; available online at https://doi.org/10.1016/j.jnt.2015.07.021

[22] F. Qi, R.-Q. Cui, C.-P. Chen, and B.-N. Guo, Some completely monotonic functions involving polygamma functions and an application, J. Math. Anal. Appl. 310 (2005), no. 1, 303-308; available online at http://dx.doi.org/10.1016/j.jmaa.2005.02.016

[23] F. Qi and B.-N. Guo, Lévy-Khintchine representation of Toader-Qi mean, Math. Inequal. Appl. 21 (2018), no. 2, 421-431; available online at https://doi.org/10.7153/ mia-2018-21-29.

[24] F. Qi and B.-N. Guo, The reciprocal of the weighted geometric mean of many positive numbers is a Stieltjes function, Quaest. Math. 41 (2018), no. 5, 653-664; available online at https: //doi.org/10.2989/16073606.2017.1396508

[25] F. Qi and W.-H. Li, Integral representations and properties of some functions involving the logarithmic function, Filomat 30 (2016), no. 7, 1659-1674; available online at https: //doi.org/10.2298/FIL1607659Q

[26] F. Qi and D. Lim, Integral representations of bivariate complex geometric mean and their applications, J. Comput. Appl. Math. 330 (2018), 41-58; available online at https://doi. org $/ 10.1016 /$ j.cam.2017.08.005

[27] F. Qi and A.-Q. Liu, Notes on complete monotonicity related to the difference of the psi and logarithmic functions, HAL preprint (2018), available online at https://hal. archives-ouvertes.fr/hal-01728682

[28] F. Qi, X.-J. Zhang, and W.-H. Li, Lévy-Khintchine representations of the weighted geometric mean and the logarithmic mean, Mediterr. J. Math. 11 (2014), no. 2, 315-327; available online at https://doi.org/10.1007/s00009-013-0311-z 
[29] F. Qi, X.-J. Zhang, and W.-H. Li, The harmonic and geometric means are Bernstein functions, Bol. Soc. Mat. Mex. (3) 23 (2017), no. 2, 713-736; available online at https: //doi.org/10.1007/s40590-016-0085-y

[30] S.-L. Qiu and M. Vuorinen, Some properties of the gamma and psi functions, with applications, Math. Comp. 74 (2005), no. 250, 723-742; available online at https://doi.org/10. 1090/S0025-5718-04-01675-8

[31] R. L. Schilling, R. Song, and Z. Vondraček, Bernstein Functions-Theory and Applications, 2nd ed., de Gruyter Studies in Mathematics 37, Walter de Gruyter, Berlin, Germany, 2012; available online at https://doi.org/10.1515/9783110269338

[32] D. V. Widder, The Laplace Transform, Princeton University Press, Princeton, 1946.

[33] S.-Q. Zhang, B.-N. Guo, and F. Qi, A concise proof for properties of three functions involving the exponential function, Appl. Math. E-Notes 9 (2009), 177-183.

(Qi) Institute of Mathematics, Henan Polytechnic University, Jiaozuo 454010, Henan, China; College of Mathematics, Inner Mongolia University for Nationalities, Tongliao 028043, Inner Mongolia, China; School of Mathematical Sciences, Tianjin Polytechnic University, Tianjin 300387, China

Email address: qifeng618@gmail.com, qifeng618@hotmail.com, qifeng618@qq.com

$U R L:$ https://qifeng618. wordpress.com

(Liu) Department of Mathematics, Sanmenxia Polytechnic, Sanmenxia 472000, Henan, CHINA

Email address: lady_smx@126.com, smxptliu@hotmail.com 